GRADIATION\&APPLICATIONS

ISSN 2466-4294 (online) | rad-journal.org

Vol. 2 | Issue 3 | pp. $233-235,2017$

doi: 10.21175/RadJ.2017.03.047

Short note

\title{
EFFECTS OF IN VIVO PROTON IRRADIATION ON MOUSE T AND B LYMPHOCYTES*
}

\author{
Flavia Novelli' ${ }^{1}$, Monia Vadrucci ${ }^{2}$, Maria Manuela Rosado ${ }^{3}$, \\ Luigi Picardi ${ }^{2}$, Eugenio Benvenuto ${ }^{4}$, Claudio Pioli ${ }^{* *}$
}

\author{
${ }^{1}$ Laboratory of Biomedical Technologies, ENEA, Rome Italy \\ ${ }^{2}$ Laboratory of Development of Particle Accelerators and Medical Applications, ENEA, Rome, Italy \\ ${ }^{3}$ Consultant in Immunology, Rome, Italy \\ 4 Laboratory of Biotechnology, ENEA, Rome, Italy
}

\begin{abstract}
One of the major problems derived from the exposure to ionizing radiation is the impairment of the immune system. The consequent immune-depression increases the risk of infections and may lead to immunemediated disorders. The intensity and duration of the immune-compromised phase and its recovery depend on the dose, dose-rate and quality of radiation. In recent years, there has been a great interest in the effects induced by protons, both for a better assessment of the health risks in astronauts exposed to solar wind and cosmic radiations and for a better understanding of their effects in radiotherapy for oncologic patients. In the present study, we investigated the effects of the in vivo exposure to 2 Gy of integral dose absorbed by medium energy proton beams on mouse lymphoid spleen cells. The TOP-IMPLART accelerator was used as proton source. Irradiations were performed in air with pulsed $(3.4 \mu \mathrm{s}, 1 \mathrm{OHz}) 27 \mathrm{MeV}$ proton beams. During the exposure, mice were anesthetized in order to keep them in the right position. Sham-exposed anesthetized age/gender/strain-matched mice were used as controls. Twenty-four hours and 1 week after irradiation, each mouse was individually analyzed for several parameters (5 mice/group). Results showed that the number of nucleated cells in the spleen was not significantly affected. Flow cytometry analyses revealed that the percentages of helper $T(C D 4)$, cytotoxic $T(C D 8)$ and B (CD19) cells within the spleen lymphocytes were not altered 24 hours after the exposure. At variance, 1 week after the exposure the frequency of $\mathrm{CD}_{4}(14 \%$ vs. $9 \%)$ and $\mathrm{CD} 19$ (37\% vs. 26\%) cells reduced. Spleen cells were stimulated with an anti-CD3 antibody and LPS to induce T cell and B cell activation, respectively. Both $T$ and $B$ cells were functionally impaired by the exposure. Twenty-four hours after irradiation, T cell proliferation was indeed reduced by $50 \%$ in exposed mice compared with controls. $B$ cells also displayed a reduced cell proliferation in response to the mitogenic stimulus (-33\%). Interestingly, 1 week after irradiation proliferative responses of $T$ and $B$ cells were still compromised. This first study allowed the conclusion that, in vivo local exposure to protons induced small changes in total spleen cell number, the frequency of $\mathrm{CD}_{4}$ and $B$ cells being reduced 1 week after the exposure. More interesting, functional responses, such as $T$ and $B$ cell proliferation were partially compromised. These effects, in spite of the limited area of exposure, were not recovered after 1 week.
\end{abstract}

Key words: Antibodies, cell proliferation, immune system, lymphocytes, protons, space flights

\section{INTRODUCTION}

During space flights and/or extravehicular activity, astronauts are exposed to several stressors having the potential to alter immune responses (microgravity, radiations, psychological stress, and others) [1]. Ionizing radiations include galactic/cosmic radiation and solar wind. Solar radiation is mainly composed of protons with a minor contribution from heavy ions and electrons. Ionizing radiation is very well known to affect several immune system parameters, primarily due to its effects on immune-hematopoietic cell survival. The effects include alterations in cell number, leukocyte subpopulation frequencies, cytokine production, and antibody production. While the large majority of these results were obtained exposing cells and animals to $\mathrm{X}$ or $\gamma$ rays, data on the effects of exposure to protons are still limited [2-7]. Weakening of the immune system might represent a serious threat for astronauts as it could expose them not only to the action of genuine pathogens but it could also allow the opportunistic pathogens or latent virus to infect the host.

Besides, there is also a great interest in understanding the effects on the immune system of the exposure to protons due to radiotherapy. Proton therapy is very promising as it allows a more focused action on targeted tumors, sparing healthy tissues. Interestingly, in recent years, systemic effects on the immune system of patients exposed to local

\footnotetext{
* The paper was presented at the Fifth International Conference on Radiation and Applications in Various Fields of Research (RAD 2017), Budva, Montenegro, 2017.

*kaudio.pioli@enea.it
} 
radiotherapy were described, resulting in both, therapeutic beneficial effects or harmful side effects [8-9].

In the present study, we investigated the effects of the in vivo exposure to 2 Gy of integral dose absorbed by medium energy proton beams on mouse lymphoid spleen cells. The proton accelerator, under implementation in the framework of the Italian TOP-IMPLART project, was used [10].

\section{MAterials AND MEthods}

The proton accelerator, which is under construction in the framework of the Italian TOP-IMPLART project, consists of a low radiofrequency $(425 \mathrm{MHz})$ and low energy $(7 \mathrm{MeV})$ injector and linear structures operating at high radio-frequency $(3 \mathrm{GHz})$ to accelerate protons to medium (35 MeV) and high energy (150 MeV). Irradiations were performed in air with pulsed $(3.4 \mu \mathrm{s}$, $10 \mathrm{~Hz}) 27 \mathrm{MeV}$ proton beams, choosing a particular setup at $100 \mathrm{~cm}$ from the accelerator to have a uniform (98.5\%) coverage of the selected area. The protons, having $24.5 \mathrm{MeV}$ residual energy, penetrate $6 \mathrm{~mm}$ in tissue. Under these conditions, lymphoid spleen cells of twelve-week-old C57BL/6 male mice adsorbed 2 Gy of integral dose by medium energy proton beams. During the exposure, mice were anesthetized to ensure immobilization and to be placed in the appropriate position for the proton beam to target the spleen. Sham-exposed mice, which underwent the same treatments as exposed mice except for the proton beam, were used as controls. Preliminary experiments were performed to measure the dimensions and position of the spleen in mice from the same lot to ensure the correct targeting of this organ.

Spleen cells from sham-exposed mice and mice exposed to proton beam 24 hours or 1 week before sacrifice (5 mice/group) were collected, counted, analyzed by flow cytometry and stimulated in vitro to induce cell proliferation. Briefly, cells were stained with fluorochrome-conjugated anti-CD4, anti-CD8 and antiCD19 antibodies (Miltenyi Biotec) to identify helper T, cytotoxic $\mathrm{T}$ and $\mathrm{B}$ cells, respectively. Optimal concentrations of antibodies and reagents were assessed in previous experiments. Fluorescence signals were collected in the log mode using a FACSCalibur (BD Biosciences). Analyses of cell populations were performed on events gated according to forward scatter/side scatter light parameters.

Spleen cells were stimulated with anti-CD3 mAb or LPS to induce T and B cell proliferation, respectively. After 48 hour stimulation, cell proliferation was assessed by adding the Cell Titer 96TM Aqueous one solution (Promega G3580) to culture plates according the producer's instructions. Six hours later, the absorbance was read at $490 \mathrm{~nm}$ using an ELISA microtiter plate reader.

Mice were individually analyzed for all of the parameters considered. All data are shown as means \pm standard error of mean (SEM) obtained by averaging the values of mice belonging to the same group. The Mann-Whitney U-test was used, with $\mathrm{p}<0.05$ as the criterion for significance for all statistical comparisons. A post hoc power calculation was completed for each 234 statistical comparison. The $\mathrm{X}^{2}$ test was applied to compare sham-exposed and exposed groups for proportions. A $\mathrm{p}<0.05$ was considered as significant.

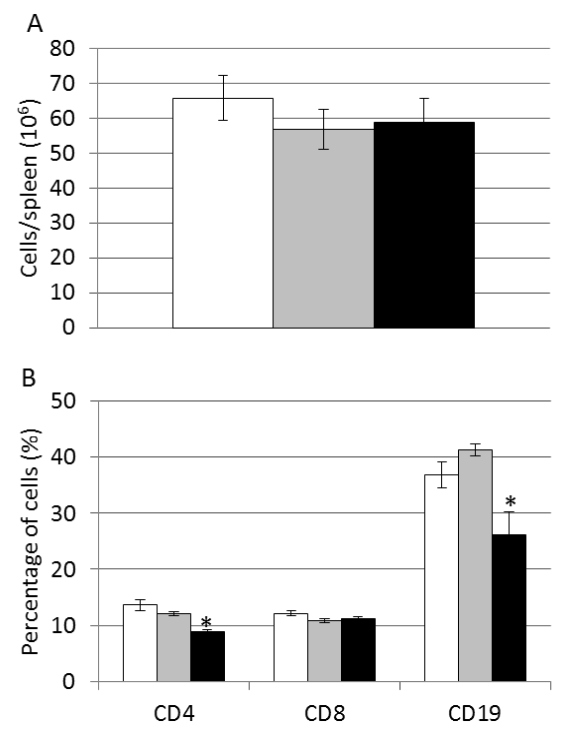

Figure 1. Spleen cell number and percentage of T- and Blymphocytes, in irradiated and sham-exposed (white columns) mice. Twenty-four hours (gray columns) and 1 week

(black columns) after exposure, spleen cells were collected, counted and analyzed by flow cytometry. A. Number of nucleated cells/spleen. B. Percentage of CD4 T, CD8 T and B

(CD19) cells. Values represent mean \pm Standard Error (5 mice/group), ${ }^{*}, \mathrm{p}<0.05$ for all comparisons.

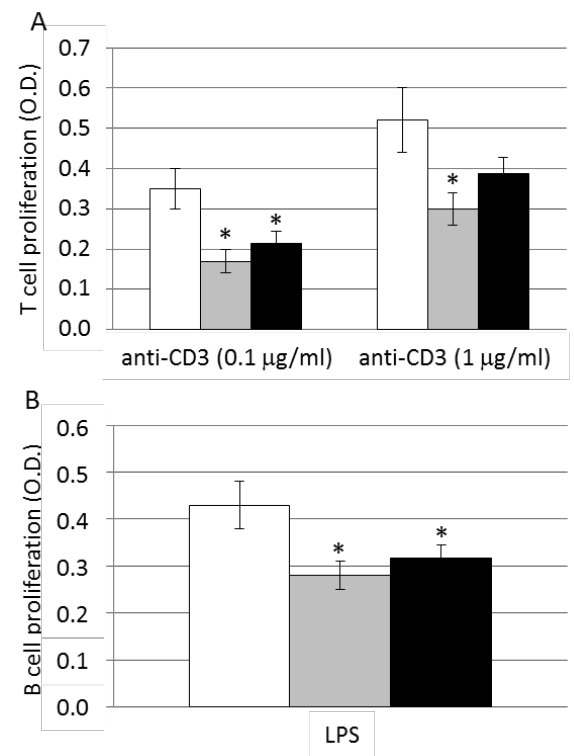

Figure 2. Twenty-four hours (gray columns) and 1 week (black columns) after irradiation, animals were sacrificed and their spleen cells were stimulated for 48 hours either with an antiCD3 monoclonal antibody at 0.1 or $1 \mu \mathrm{g} / \mathrm{ml}$ (A) or with LPS at $1 \mu \mathrm{g} / \mathrm{ml}$ (B). Spleen cells from sham-exposed mice (white columns) were used as controls. Values represent mean \pm Standard Error (5 mice/group); *, p<0.05. 


\section{RESULTS}

Results showed that the number of nucleated cells in the spleen was not significantly reduced 24 hours and 1 week after the local exposure to $2 \mathrm{~Gy}$ (Fig. 1A). Flow cytometry analyses revealed that the percentages of helper T (CD4), cytotoxic T (CD8) and B (CD19) cells within the spleen lymphocytes were not altered 24 hours after the exposure. At variance, 1 week after the exposure the frequency of CD4 (14\% vs. 9\%) and CD19 (37\% vs. 26\%) cells was reduced (Fig. 1B).

Spleen cells were stimulated with an anti-CD3 monoclonal antibody to induce $\mathrm{T}$ cell activation. Results showed that the exposure reduced the $\mathrm{T}$ cell proliferative response to both optimal $(1 \mu \mathrm{g} / \mathrm{ml})$ and sub-optimal $(0.1 \mu \mathrm{g} / \mathrm{ml})$ concentrations of anti-CD3 antibody. One week after irradiation the $\mathrm{T}$ cell proliferation was still compromised, showing only a partial recover (Fig. 2A) as compared to proliferative response of $\mathrm{T}$ cells from sham-exposed mice.

Spleen cells were also stimulated with LPS (lipopolysaccharide, $1 \mu \mathrm{g} / \mathrm{ml}$ ) to induce B cell proliferation. Results showed that the $\mathrm{B}$ cell proliferative response 24 hours and 1 week after the exposure was also significantly reduced (Fig. 2B).

\section{CONCLUSIONS}

With this first explorative study we can conclude that in vivo local exposure to protons induces small changes in total spleen cell number, the frequency of CD4 and B cells being reduced 1 week after the exposure. More interesting, functional responses, such as $\mathrm{T}$ and $\mathrm{B}$ cell proliferation were partially compromised. These effects, in spite of the limited area of exposure, were not recovered after 1 week. Additional experiments are ongoing to investigate the effects of protons at further time points after the irradiation and on other immunologic parameters including cytokine secretion and antibody production.

Acknowledgement: The present work was partially supported by the Italian Space Agency (ASI) project BIOxTREME. The proton accelerator is realized with the support of the TOIMPLART project from Regione Lazio (Italy).

\section{REFERENCES}

1. N. Gueguinou et al., "Could spaceflight-associated immune system weakening preclude the expansion of human presence beyond earth's orbit?” J. Leukoc. Biol., vol. 86, no. 5, pp. 1027 - 1038, Nov. 2009.

DOI: $10.1189 /$ jlb.0309167

PMid: 19690292

2. D. Frasca et al., "Hematopoietic reconstitution after lethal irradiation and bone marrow transplantation: effects of different hematopoietic cytokines on the recovery of thymus, spleen and blood cells," Bone Marrow Transplant., vol. 25, no. 4, pp. 427 - 433, Feb. 2000.

DOI: $10.1038 /$ sj.bmt. 1702169

PMid: 10723587

3. D. Frasca et al., "Use of hematopoietic cytokines to accelerate the recovery of the immune system in irradiated mice," Exp. Hematol., vol. 25, no. 11, pp. 1167 - 1171, Oct. 1997. PMid: 9328453

4. D. Frasca et al., "IL-11 synergizes with IL-3 in promoting the recovery of the immune system after irradiation," Int. Immunol., vol. 8, no. 11, pp. $1651-1657$, Nov. 1996.

DOI: $10.1093 /$ intimm/8.11.1651 PMid: 8943559

5. P. Uma Devi, "Radiosensitivity of the developing haemopoietic system in mammals and its adult consequences: animal studies," Br. J. Radiol., vol. 76, no. 906, pp. $366-372$, Jun. 2003

DOI: $10.1259 / \mathrm{bjr} / 42623440$

PMid: 12814921

6. K. Manda et al., "Effects of ionizing radiation on the immune system with special emphasis on the interaction of dendritic and T cells," Front. Oncol., vol. 2, no. 102. Aug. 2012.

DOI: 10.3389 /fonc.2012.00102

7. J. H. Ware et al., "Effects of proton radiation dose, dose rate and dose fractionation on hematopoietic cells in mice," Radiat. Res., vol. 174, no. 3, pp. $325-330$, Sep. 2010.

DOI: $10.1667 / R R 1979.1$

PMid: 20726731 PMCid: PMC 3405897

8. S. C. Formenti et al., "Systemic effects of local radiotherapy," Lancet oncol., vol. 10, no. 7, pp. $718-726$, Jul 2009. DOI: 10.1016/S1470-2045(09)70082-8

9. F. Rodel et al., "Contribution of the immune system to bystander and non-targeted effects of ionizing radiation," Cancer Lett., vol. 356, no. 1, pp. $105-113$, Jan. 2015.

DOI: 10.1016/j.canlet.2013.09.015

PMid: 24139966

10. M. Vadrucci et al., "Calibration of GafChromic EBT3 for absorbed dose measurements in $5 \mathrm{MeV}$ proton beam and (60)Co gamma-rays," Med. Phys., vol. 42, no. 8, pp. $4678-4684$, Aug. 2015.

DOI: $10.1118 / 1.4926558$

PMid: 26233195 